Article

\title{
Fixed Points of Eventually $\Delta$-Restrictive and $\Delta(\epsilon)$-Restrictive Set-Valued Maps in Metric Spaces
}

\author{
Pradip Debnath ${ }^{1, *(1)}$ and Manuel de La Sen ${ }^{2, *}$ \\ 1 Department of Applied Science and Humanities, Assam University, Silchar, Cachar, Assam 788011, India \\ 2 Institute of Research and Development of Processes, University of the Basque Country, \\ Campus of Leioa, 48940 Leioa (Bizakaia), Spain \\ * Correspondence: pradip.debnath@aus.ac.in or debnath.pradip@yahoo.com (P.D.); \\ manuel.delasen@ehu.eus (M.d.L.S.); Tel.: +91-8575158469 (P.D.)
}

Received: 22 December 2019; Accepted: 6 January 2020 ; Published: 8 January 2020

check for updates

\begin{abstract}
The symmetry concept is an intrinsic property of metric spaces as the metric function generalizes the notion of distance between two points. There are several remarkable results in science in connection with symmetry principles that can be proved using fixed point arguments. Therefore, fixed point theory and symmetry principles bear significant correlation between them. In this paper, we introduce the new definition of the eventually $\Delta$-restrictive set-valued map together with the concept of $p$-orbital continuity. Further, we introduce another new concept called the $\Delta(\epsilon)$-restrictive set-valued map. We establish several fixed point results related to these maps and proofs of these results also provide us with schemes to find a fixed point. In a couple of results, the stronger condition of compactness of the underlying metric space is assumed. Some results are illustrated with examples.
\end{abstract}

Keywords: Fixed point; metric space; set-valued map; $\Delta(\epsilon)$-restrictive map; eventually $\Delta$-restrictive map

\section{Preliminaries}

Markin [1] and Nadler [2] initiated the investigation of fixed points for set-valued maps in a metric space (MS). For some significant results on fixed points of set-valued maps and their applications, we refer to the works of Assad and Kirk [3], Jleli et al. [4], Kirk and Shahzad [5], Chifu and Petrusel [6], Qawaqneh et al. [7], Pathak [8], Debnath and Sen [9].

The Pompeiu-Hausdorff metric is defined in the following manner for the MS $(X, \delta)$, where $X$ is a non-empty set and $\delta$ is the concerned metric. We denote this metric by $\mathcal{P} \mathcal{H}$.

By $\Gamma(X)$ we denote the class of all nonempty closed and bounded subsets of $X$. Define the function $\mathcal{P} \mathcal{H}: \Gamma(X) \times \Gamma(X) \rightarrow[0, \infty)$ by

$$
\mathcal{P H}(U, V)=\max \left\{\sup _{\xi \in V} \Delta(\xi, U), \sup _{\eta \in U} \Delta(\eta, V)\right\}, \text { for all } U, V \in \Gamma(X),
$$

where $\Delta(\eta, V)=\inf _{\xi \in V} \delta(\eta, \xi)$. Then $(\Gamma(X), \mathcal{P H})$ is an MS induced by $\delta$ and is called the Pompeiu-Hausdorff MS.

Definition 1. [2] Let $R: X \rightarrow \Gamma(X)$ be a set-valued map. $\mu \in X$ is called a fixed point of $R$ if $\mu \in R \mu$.

Remark 1. 1. It is to be noted that in the $M S(\Gamma(X), \mathcal{P H}), \mu \in X$ is a fixed point of $R$ if and only if $\Delta(\mu, R \mu)=0$. The function $\Delta$ plays a special role in the current paper.

2. It is well known that the metric function $\delta: X \times X \rightarrow[0, \infty)$ is continuous in the sense that if $\left\{\mu_{n}\right\},\left\{v_{n}\right\}$ are two sequences in $X$ with $\left(\mu_{n}, v_{n}\right) \rightarrow(\mu, v)$ for some $\mu, v \in X$, as $n \rightarrow \infty$, then $\delta\left(\mu_{n}, v_{n}\right) \rightarrow \delta(\mu, v)$ 
as $n \rightarrow \infty$. It follows that the function $\Delta$ is continuous in the sense that if $\mu_{n} \rightarrow \mu$ as $n \rightarrow \infty$, then $\Delta\left(\mu_{n}, U\right) \rightarrow \Delta(\mu, U)$ as $n \rightarrow \infty$ for any $U \subseteq X$

Some important relations among $\delta, \Delta$ and $\mathcal{P} \mathcal{H}$, which are relevant to the sequel, may be found in $[10,11]$. For some well known modern monographs in fixed point theory of multivalued mappings we refer to [12-14].

Continuity of a set-valued map in an MS is defined in terms of upper semi-continuity and lower semi-continuity [15]. We define the following concept of $\mathcal{P} \mathcal{H}$-continuity.

Definition 2. Let $(X, \delta)$ be an MS. A set-valued map $R: X \rightarrow \Gamma(X)$ is said to be $\mathcal{P} \mathcal{H}$-continuous at a point $\mu_{0}$, if for each sequence $\left\{\mu_{n}\right\} \subset X$, such that $\lim _{n \rightarrow \infty} \delta\left(\mu_{n}, \mu_{0}\right)=0$, we have $\lim _{n \rightarrow \infty} \mathcal{P} \mathcal{H}\left(R \mu_{n}, R \mu_{0}\right)=0$ (i.e., if $\mu_{n} \rightarrow \mu_{0}$, then $R \mu_{n} \rightarrow R \mu_{0}$ as $n \rightarrow \infty$ ).

Or equivalently, $R$ is said to be $\mathcal{P H}$-continuous at a point $\mu_{0}$, if for every $\epsilon>0$, there exists $\lambda>0$ such that $\mathcal{P} \mathcal{H}\left(R \mu, R \mu_{0}\right)<\epsilon$, whenever $\delta\left(\mu, \mu_{0}\right)<\lambda$.

Definition 3. [2] Let $R: X \rightarrow \Gamma(X)$ be a set-valued map. $R$ is said to be a set-valued contraction if $\mathcal{P H}(R \mu, R v) \leq \lambda \delta(\mu, v)$ for all $\mu, v \in X$, where $\lambda \in[0,1)$.

Remark 2. 1. $R$ is $\mathcal{P H}$-continuous on a subset $S$ of $X$ if it is continuous on every point of $S$.

2. If $R$ is a set-valued contraction, then it is $\mathcal{P H}$-continuous.

Below we give an example of $\mathcal{P} \mathcal{H}$-continuous map.

Example 1. Consider $X=\mathbb{R}$ with usual metric $\delta(\mu, v)=|\mu-v|$.

Define $R: X \rightarrow \Gamma(X)$ by

$$
R(\mu)=[\mu, \mu+1] \text { for all } \mu \in \mathbb{R} .
$$

Then $\mathcal{P H}(R \mu, R v)=|\mu-v|$. Let $\epsilon>0$ and $\lambda=\frac{\epsilon}{2}$. Therefore, $\mathcal{P H}(R \mu, R v)<\epsilon$ whenever $|\mu-v|<\lambda$. Hence $R$ is $\mathcal{P} \mathcal{H}$-continuous.

Orbital sequences play an important role in the study of fixed points for set-valued or multi-valued maps (see [16,17]).

Definition 4. Let $(X, \delta)$ be an $M S$ and $R: X \rightarrow \Gamma(X)$ a set-valued map. An R-orbital (or, simply orbital) sequence of $R$ at a point $\mu \in X$ is a set $O(\mu, R)$ of points in $X$ defined by $O(\mu, R)=\left\{\mu_{0}=\mu, \mu_{n+1} \in\right.$ $\left.R \mu_{n}, n=0,1,2, \ldots\right\}$.

Edelstein [18,19] studied the implications of several modifications in the assumptions of the famous Banach contraction principle.

In [18], the concept of $\epsilon$-contractive mappings was put forward and the existence of fixed and periodic points for such maps were investigated. Also, the uniqueness of fixed points were established by assuming compactness of the metric space. Further, in [19], fixed points of non-expansive mappings on Banach spaces were investigated.

Inspired by the work of Edelstein on fixed and periodic points, in the current paper we introduce the new notions of eventually $\Delta$-restrictive and $\Delta(\epsilon)$-restrictive set-valued maps in metric spaces. Roughly, the restrictive conditions of our theorems may be termed as Edelstein-type conditions for set-valued maps. For some relevant Edelstein-type results, we refer to the works of Aydi et al. [20], Bailey [21]. The main motivation of the present study is to provide an alternative approach for the investigation of fixed points of certain class of set-valued mappings by developing some new hypothesis which we call restrictive conditions.

The main results of this paper are divided into two sections. In the first section we discuss the fixed points of eventually $\Delta$-restrictive set-valued maps while in the later we study the same for 
$\Delta(\epsilon)$-restrictive set-valued maps. In each section we present two results and in the second result of each section, compactness of the MS is assumed.

\section{Eventually $\Delta$-Restrictive Set-Valued Map}

Now we are ready to present the main results.

In this section, we first define an eventually $\Delta$-restrictive set-valued map and then introduce the notion of $p$-orbital continuity.

Definition 5. Let $(X, \delta)$ be an MS. A set-valued map $R: X \rightarrow \Gamma(X)$ is said to be eventually $\Delta$-restrictive if whenever $\Delta(\mu, R \mu)>0$ for some $\mu \in X$, then there exists $k \in \mathbb{N}$ such that $\Delta\left(\mu_{n}, R \mu_{n}\right)<\Delta(\mu, R \mu)$ for all $n \geq k$, where $\mu_{n} \in O(\mu, R)$.

Definition 6. Let $(X, \delta)$ be an MS. A set-valued map $R: X \rightarrow \Gamma(X)$ is said to be p-orbitally continuous if whenever a subsequence $\left\{\mu_{n_{i}}\right\}$ of a sequence $\left\{\mu_{n}\right\}$ converges to some $\mu \in X$, i.e., if $\mu_{n_{i}} \rightarrow \mu$, then for any fixed $p \in \mathbb{N}, \mu_{n_{i}+p} \rightarrow \mu_{p}$ and $R \mu_{n_{i}+p} \rightarrow R \mu_{p}$, where $\mu_{p}$ is the $p$-th term of the $R$-orbital sequence $O(\mu, R)$.

Theorem 1. Let $(X, \delta)$ be an MS and $R: X \rightarrow \Gamma(X)$ be an eventually $\Delta$-restrictive and p-orbitally continuous set-valued map. If for some $\mu_{0} \in X$, the orbital sequence $O\left(\mu_{0}, R\right)$ of $R$ has a subsequence $\left\{\mu_{n_{i}}\right\}$ converging to $\theta$, then $\theta$ is a fixed point of $R$.

Proof. Without the loss of generality, we assume that $\mu_{n+1} \neq \mu_{n}$ and also $\mu_{n} \notin \operatorname{Fix}(R)$ for all $n \in \mathbb{N}$, for otherwise we trivially have a fixed point.

Since $\lim _{i \rightarrow \infty} \mu_{n_{i}}=\theta$ and $R$ is $p$-orbitally continuous, for any fixed positive integer $p$, we have $\lim _{i \rightarrow \infty} \delta\left(\mu_{n_{i}+p}, \mu_{n_{i}+p+1}\right)=\delta\left(\mu_{p}, \mu_{p+1}\right)$.

Let $\theta \notin R \theta$. Then $\Delta(\mu, R \mu)>0$. Since $R$ is eventually $\Delta$-restrictive, there exists $r \in \mathbb{N}$ such that $\Delta\left(\theta_{r}, R \theta_{r}\right)<\Delta(\theta, R \theta)$.

Let $\epsilon_{0}=\frac{1}{2}\left[\Delta(\theta, R \theta)-\Delta\left(\theta_{r}, R \theta_{r}\right)\right]>0$.

Since $\lim _{i \rightarrow \infty} \Delta\left(\mu_{n_{i}+r}, R \mu_{n_{i}+r}\right)=\Delta\left(\theta_{r}, R \theta_{r}\right)$ (for $\Delta$ is continuous and $R$ is $p$-orbitally continuous), for arbitrary $\epsilon>0$, there exists a sufficiently large $n_{i}=q$ such that $\Delta\left(\mu_{q+r}, R \mu_{q+r}\right)<\Delta\left(\theta_{r}, R \theta_{r}\right)+\epsilon$.

For $\epsilon=\epsilon_{0}$, we have $\Delta\left(\mu_{q+r}, R \mu_{q+r}\right)<\frac{1}{2}\left[\Delta(\theta, R \theta)+\Delta\left(\theta_{r}, R \theta_{r}\right)\right]$.

Since $\Delta\left(\mu_{n}, R \mu_{n}\right)>0$ (for $\mu_{n+1} \neq \mu_{n}$ and $\mu_{n} \notin \operatorname{Fix}(R)$ ) for all $n \in \mathbb{N}$ and $R$ eventually $\Delta$-restrictive, there exists a non-negative integer $k$ such that

$\Delta\left(\mu_{n+q+r}, R \mu_{n+q+r}\right)<\Delta\left(\mu_{q+r}, R \mu_{q+r}\right)$ for all $n \geq k$, where $\mu_{n+q+r} \in O\left(\mu_{q+r}, n\right)$, i.e., $\Delta\left(\mu_{n}, R \mu_{n}\right)<\Delta\left(\mu_{q+r}, R \mu_{q+r}\right)$ for all $n \geq k+q+r$, where $\mu_{n} \in O\left(\mu_{q+r}, n\right)$.

So we obtain that

$\Delta\left(\mu_{n}, R \mu_{n}\right)<\frac{1}{2}\left[\Delta(\theta, R \theta)+\Delta\left(\theta_{r}, R \theta_{r}\right)\right]$ for all $n \geq k+q+r$.

Thus $\Delta\left(\mu_{n_{i}}, R \mu_{n_{i}}\right)<\frac{1}{2}\left[\Delta(\theta, R \theta)+\Delta\left(\theta_{r}, R \theta_{r}\right)\right]$ for all $n_{i} \geq k+q+r$.

Consequently, using the facts that $\Delta$ is continuous, $R$ is $p$-orbitally continuous and the hypothesis that $\lim _{i \rightarrow \infty} \mu_{n_{i}}=\theta$, we obtain

$$
\begin{aligned}
\Delta(\theta, R \theta) & =\lim _{i \rightarrow \infty} \Delta\left(\mu_{n_{i}}, R \mu_{n_{i}}\right) \\
& \leq \frac{1}{2}\left[\Delta(\theta, R \theta)+\Delta\left(\theta_{r}, R \theta_{r}\right)\right] .
\end{aligned}
$$

Hence $\Delta(\theta, R \theta) \leq \Delta\left(\theta_{r}, R \theta_{r}\right)$, which is a contradiction to the choice of $r$. Therefore, $\Delta(\theta, R \theta)=0$, i.e., $\theta \in R \theta$.

Example 2. Consider the set $X=[0,4]$ with usual metric $\delta(\mu, v)=|\mu-v|$ for all $\mu, v \in X$. Also define $\xi: X \rightarrow X$ such that 


$$
\xi(\mu)= \begin{cases}\frac{\mu}{2}+2, & \text { if } 0 \leq \mu<2 \\ 4-\frac{\mu}{2}, & \text { if } 2 \leq \mu \leq 4 .\end{cases}
$$

Define $R: X \rightarrow \Gamma(X)$ by $R \mu=\{2\} \cup\{\xi(\mu)\}$ for each $\mu \in X$. If Fix $(R)$ denotes the set of fixed points of $R$, then it is easy to see that Fix $(R)=\left\{2, \frac{8}{3}\right\}$.

It's easy to check that the map $R$ is eventually contractive and p-orbitally continuous.

Further, construct the orbital sequence $\left\{\mu_{n}\right\}_{n=0}^{\infty}$ such that $\mu_{n+1}=4-\frac{\mu_{n}}{2}$ with $\mu_{0}=4$. So, we have $\left\{\mu_{n}\right\}_{n=0}^{\infty}=\left\{\mu_{0}=4, \mu_{1}=2, \mu_{2}=3, \mu_{3}=\frac{5}{2}, \mu_{4}=\frac{11}{4}, \mu_{5}=\frac{21}{8}, \mu_{6}=\frac{43}{16}, \mu_{7}=\frac{85}{32}, \ldots\right\}$. Clearly, $\mu_{n+1} \in R \mu_{n}$ for all $n=0,1,2, \ldots$.

Also $\left\{\mu_{n}\right\}_{n=0}^{\infty}$ has a subsequence $\left\{\mu_{3}=\frac{5}{2}, \mu_{5}=\frac{21}{8}, \mu_{7}=\frac{85}{32}, \mu_{9}=\frac{341}{128}, \ldots\right\}$, which is monotone increasing with supremum $\frac{8}{3}$ and thus converges to $\frac{8}{3}$.

Thus all conditions of Theorem 1 are satisfied and we can see that $\frac{8}{3}$ is a fixed point of $R$. In fact $\operatorname{Fix}(R)=\left\{2, \frac{8}{3}\right\}$.

Next result is a variant of the previous theorem, where stronger conditions such as compactness of the underlying MS and $\mathcal{P H}$-continuity of the map is assumed.

Theorem 2. Let $(X, \delta)$ be a compact $M S$ and $R: X \rightarrow \Gamma(X)$ a PH-continuous set-valued map such that for each $\mu \in X$, there exists $n \in \mathbb{N}$ for which $\Delta(\mu, R \mu)>0$ implies $\Delta\left(\mu_{n}, R \mu_{n}\right)<\Delta(\mu, R \mu)$, where $\mu_{n}$ is the $n$-th term of the $R$-orbital sequence $O(\mu, R)$. Then $R$ has a fixed point.

Proof. Define the function $\psi: X \rightarrow[0, \infty)$ by $\psi(\mu)=\Delta(\mu, R \mu)$. Since $\delta$ and consequently $\Delta$ both are continuous, $\psi$ is continuous as well. Thus $\psi$ attains its minimum value at some point, say at $\theta \in X$.

Now if $\Delta(\theta, R \theta)>0$, then by hypothesis, there exists $n \in \mathbb{N}$ for which $\Delta\left(\theta_{n}, R \theta_{n}\right)<\Delta(\theta, R \theta)$. This implies $\psi\left(\theta_{n}\right)<\psi(\theta)$, which is a contradiction to the choice of $\theta$. Hence $\Delta(\theta, R \theta)=0$, i.e., $\theta \in R \theta$.

\section{3. $\Delta(\epsilon)$-Restrictive Set-Valued Map}

In this section, we introduce the new concept of the $\Delta(\epsilon)$-restrictive set-valued map and establish the corresponding fixed point results.

Definition 7. Let $(X, \delta)$ be an MS and $R: X \rightarrow \Gamma(X)$ be a set-valued map. $R$ is said to be $\Delta(\epsilon)$-restrictive if there exists $\epsilon>0$ such that whenever $0<\Delta(\gamma, R \gamma)<\epsilon$ for some $\gamma \in X$, then $\Delta(\mu, R \mu)<\Delta(\gamma, R \gamma)$ for all $\mu \in R \gamma$.

Theorem 3. Let $(X, \delta)$ be an $M S$ and the set-valued map $R: X \rightarrow \Gamma(X)$ be $\mathcal{P} \mathcal{H}$-continuous and $\Delta(\epsilon)$-restrictive. If there exists $\mu_{0} \in X$ such that the $R$-orbital sequence $O\left(\mu_{0}, R\right)$, contains a convergent subsequence $\mu_{n_{i}}$ which converges to $\theta$, then $\theta$ is a fixed point of $R$.

Proof. Without the loss of generality, we assume that $\mu_{n+1} \neq \mu_{n}$ and also $\mu_{n} \notin \operatorname{Fix}(R)$ for all $n \in \mathbb{N}$, for otherwise we trivially have a fixed point.

Since $\mu_{n_{i}}$ is a convergent subsequence of $\mu_{n}$, given $\epsilon>0$, there exist integers $n_{p}$ and $n_{p+1}$ such that $\delta\left(\mu_{n_{p}}, \mu_{n_{p+1}}\right)<\epsilon$. Thus we have $\Delta\left(\mu_{n_{p}}, R \mu_{n_{p}}\right)<\epsilon$ (since $\left.\mu_{n_{p+1}} \in R \mu_{n_{p}}\right)$. Further, $\mu_{n_{p}} \notin R \mu_{n_{p}}$ and therefore,

$$
\Delta\left(\mu_{n_{p+1}}, R \mu_{n_{p+1}}\right)<\Delta\left(\mu_{n_{p}}, R \mu_{n_{p}}\right) \text { (since } R \text { is } \Delta(\epsilon) \text {-restrictive). }
$$

Thus the sequence $\left\{\Delta\left(\mu_{m}, R \mu_{m}\right)\right\}_{m=n_{p}}^{\infty}$ is a strictly decreasing sequence of real numbers and hence is convergent. 
Now

$$
\begin{aligned}
\mu_{n_{p+1}} \in R \mu_{n_{p}} \\
\Rightarrow \Delta\left(\mu_{n_{p+1}}, R \mu_{n_{p}}\right)=0 \\
\Rightarrow \lim _{p \rightarrow \infty} \Delta\left(\mu_{n_{p+1}}, R \mu_{n_{p}}\right)=0 \\
\Rightarrow \Delta(\eta, R \theta)=0\left(\text { for some } \eta \in M, \text { since } R \text { is } \mathcal{P} \mathcal{H} \text {-continuous and } \mu_{n_{p}} \rightarrow \theta \text { as } p \rightarrow \infty\right. \\
\left.\quad \text { by the hypothesis that } \mu_{n_{i}} \rightarrow \theta\right) \\
\Rightarrow \mu_{n_{p+1}} \rightarrow \eta \text { and } \eta \in R \theta .
\end{aligned}
$$

Again $\lim _{p \rightarrow \infty} \Delta\left(\mu_{n_{p}}, R \mu_{n_{p}}\right)=\Delta(\theta, R \theta)<\epsilon$ and $\lim _{p \rightarrow \infty} \Delta\left(\mu_{n_{p+1}}, R \mu_{n_{p+1}}\right)=\Delta(\eta, R \eta)$.

Now the sequence $\left\{\Delta\left(\mu_{l}, R \mu_{l}\right)\right\}_{l=n_{p+1}}^{\infty}$ is a subsequence of the convergent sequence $\left\{\Delta\left(\mu_{m}, R \mu_{m}\right)\right\}_{m=n_{p}}^{\infty}$ and hence converges to the same limit.

Thus we have $\Delta(\theta, R \theta)=\Delta(\eta, R \eta)$.

If $\theta \notin R \theta$, since $\eta \in R \theta$ and $\Delta(\theta, R \theta)<\epsilon$, we would have $\Delta(\eta, R \eta)<\Delta(\theta, R \theta)$, which is a contradiction. Hence we must have $\theta \in R \theta$.

Example 3. Consider the set $X=[0,1]$ with $\xi: X \rightarrow X$ such that

$$
\xi(\mu)= \begin{cases}\frac{\mu}{2}+\frac{1}{2}, & \text { if } 0 \leq \mu<\frac{1}{2} \\ 1-\frac{\mu}{2}, & \text { if } \frac{1}{2} \leq \mu \leq 1 .\end{cases}
$$

Define $R: X \rightarrow \Gamma(X)$ by $R \mu=\{0\} \cup\{\xi(\mu)\}$ for each $\mu \in X$. It is easy to see that Fix $(R)=\left\{0, \frac{2}{3}\right\}$.

Let $v \notin R v$. Then it's a routine verification to check that the map $R$ is $\epsilon$-restrictive (with $\epsilon=1$ ) and that if $\mu \in R v$, we have

$$
\Delta(\mu, R \mu)<\Delta(\nu, R v) .
$$

Further, construct the sequence $\left\{\mu_{n}\right\}_{n=0}^{\infty}$ such that $\mu_{n+1}=1-\frac{\mu_{n}}{2}$, where $\mu_{0}=1$. So, we have $\left\{\mu_{n}\right\}_{n=0}^{\infty}=$ $\left\{\mu_{0}=1, \mu_{1}=\frac{1}{2}, \mu_{2}=\frac{3}{4}, \mu_{3}=\frac{5}{8}, \mu_{4}=\frac{11}{16}, \mu_{5}=\frac{21}{32}, \mu_{6}=\frac{43}{64}, \mu_{7}=\frac{85}{129}, \mu_{8}=\frac{171}{256}, \ldots\right\}$. Clearly, $\mu_{n+1} \in R \mu_{n}$ for all $n=0,1,2, \ldots$

Now, $\left\{\mu_{n}\right\}_{n=0}^{\infty}$ has a subsequence $\left\{\mu_{2}=\frac{3}{4}, \mu_{4}=\frac{11}{16}, \mu_{6}=\frac{43}{64}, \mu_{8}=\frac{171}{256}, \ldots\right\}$, which is monotone decreasing with infimum $\frac{2}{3}$ and hence converges to $\frac{2}{3}$.

Thus all conditions of Theorem 3 are satisfied and we can see that $\frac{2}{3}$ is a fixed point of $R$.

Again the next result is a variant of Theorem 3 where a stronger condition that the MS is compact is assumed. Also, a condition analogous to $\Delta(\epsilon)$-restrictiveness on $R$ has been imposed.

Theorem 4. Let $(X, \delta)$ be a compact $M S$ and $R: X \rightarrow \Gamma(X)$ a $\mathcal{P} \mathcal{H}$-continuous set-valued map such that for each $\mu \in X$ and $\epsilon>0$, there exists $n \in \mathbb{N}$ for which $0<\Delta(\mu, R \mu)<\epsilon$ implies $\Delta\left(\mu_{n}, R \mu_{n}\right)<\Delta(\mu, R \mu)$, where $\mu_{n}$ is the $n$-th term of the orbital sequence $O(\mu, R)$. Then $R$ has a fixed point.

Proof. Let $\mu_{0} \in X$. Since $X$ is compact, the orbital sequence $\left\{\mu_{n}\right\}=O\left(\mu_{0}, n\right)$ has a limit point. So, given $\epsilon>0$, there exists $p \in \mathbb{N}$ such that $0<\Delta\left(\mu_{p}, R \mu_{p}\right)<\epsilon$.

Define the function $\psi: X \rightarrow[0, \infty)$ by $\psi(\mu)=\Delta(\mu, R \mu)$. Since $\delta$ and consequently $\Delta$ both are continuous, $\psi$ is continuous as well. Thus $\psi$ attains its minimum value at some point, say at $\theta \in X$.

Now $\psi(\theta)=\Delta(\theta, R \theta)=\min \{\Delta(\mu, R \mu): \mu \in X\} \leq \Delta\left(\mu_{p}, R \mu_{p}\right)<\epsilon$.

If $\Delta(\theta, R \theta)>0$, then by hypothesis, there exists $k \in \mathbb{N}$ such that $\Delta\left(\theta_{k}, R \theta_{k}\right)<\Delta(\theta, R \theta)$. This implies $\psi\left(\theta_{k}\right)<\psi(\theta)$, which is a contradiction to the choice of $\theta$. Hence $\Delta(\theta, R \theta)=0$, i.e., $\theta \in R \theta$. 


\section{Conclusions}

In this paper, we study fixed points of certain set-valued maps by introducing new conditions with respect to the function $\Delta$ in an MS. We call these new conditions as restrictive conditions. Our restrictive conditions are roughly analogous to those of Edelstein-type contractive conditions.

The main contribution of this article is the introduction of two new concepts called the eventually $\Delta$-restrictive and $\Delta(\epsilon)$-restrictive set-valued maps. In each section, one main result is presented together with another result as variant by imposing stronger condition of compactness of the MS. Our new definitions and results bring a different perspective to fixed point theory in the sense that they are applicable to a new class of mappings and provide an alternative approach to investigate the existence of their fixed points as suggested in some of the examples.

Study of common fixed points for similar type of restrictive set-valued maps and their applications is a suggested future work.

Author Contributions: P.D. contributed in Conceptualization, Investigation, Methodology and Writing the original draft; M.d.L.S. contributed in Investigation, Writing and Editing, Funding Acquisition for APC. All authors have read and agreed to the published version of the manuscript.

Acknowledgments: Research of the first author P. Debnath is supported by UGC (Ministry of HRD, Govt. of India) through UGC-BSR Start-Up Grant vide letter No. F.30-452/2018(BSR) dated 12 Feb 2019. The author M. de La Sen acknowledges the Grant IT 1207-19 from Basque Government. The authors express their gratitude to the learned referees for careful reading and valuable comments towards improvement of the manuscript.

Funding: This research received no other external funding.

Conflicts of Interest: The authors declare no conflict of interest.

\section{References}

1. Markin, J.T. A fixed point theorem for set- valued mappings. Bull. Amer. Math. Soc. 1968, 74, 639-640. [CrossRef]

2. Nadler, S.B. Multi-valued contraction mappings. Pac. J. Math. 1969, 30, 475-488. [CrossRef]

3. Assad, N.A.; Kirk, W.A. Fixed point theorems for set-valued mappings of contractive type. Pac. J. Math. 1972, 43, 553-561. [CrossRef]

4. Jleli, M.; Samet, B.; Vetro, C.; Vetro, F. Fixed points for multivalued mappings in $b$-metric spaces. Abstr. Appl. Anal. 2015, 718074. [CrossRef]

5. Kirk, W.A.; Shahzad, N. Fixed Point Theory in Distance Spaces; Springer: Berlin, Germany, 2014.

6. Chifu, C.; Petrusel, G. Fixed point results for multivalued Hardy-Rogers contractions in $b$-metric spaces. Filomat 2017, 31, 2499-2507. [CrossRef]

7. Qawaqneh, H.; Noorani, M.S.M.; Shatanawi, W.; Aydi, H. Fixed point results for multi-valued contractions in $b$-metric spaces and an application. Mathematics 2019, 7, 132. [CrossRef]

8. Pathak, H.K. An Introduction to Nonlinear Analysis and Fixed Point Theory; Springer: Boston, MA, USA, 2018.

9. Debnath, P.; de La Sen, M. Set-valued interpolative Hardy-Rogers and set-valued Reich-Rus-Ciric-type contractions in $b$-metric spaces. Mathematics 2019, 7, 849. [CrossRef]

10. Boriceanu, M.; Petrusel, A.; Rus, I. Fixed point theorems for some multivalued generalized contraction in b-metric spaces. Int. J. Math. Stat. 2010, 6, 65-76.

11. Czerwik, S. Nonlinear set-valued contraction mappings in b-metric spaces. Atti Sem. Mat. Univ. Modena 1998, 46, 263-276.

12. Hu, S.; Papageorgiou, N.S. Handbook of Multivalued Analysis; Springer: Boston, MA, USA, 2000.

13. Gorniewicz, L. Topological Fixed Point Theory of Multivalued Mappings; Springer: Berlin, Germany, 2006.

14. Afif, B.A.; O'Regan, D. Topological Fixed Point Theory for Singlevalued and Multivalued Mappings and Applications; Springer: Cham, Switzerland, 2016.

15. Geletu, A. Introduction to Topological Spaces and Set-Valued Maps: Lecture Notes in Math; Ilmenau University of Technology: Ilmenau, Germany, 2006.

16. Berinde, M.; Berinde, V. On a general class of multi-valued weakly picard mappings. J. Math. Anal. Appl. 2007, 326, 772-782. [CrossRef] 
17. Daffer, P.Z.; Kaneko, H. Fixed points of generalized contractive multi-valued mappings. J. Math. Anal. Appl. 1995, 192, 655-666. [CrossRef]

18. Edelstein, M. On fixed and periodic points under contractive mappings. J. Lond. Math. Soc. 1962, 37, 74-79. [CrossRef]

19. Edelstein, M. On non-expansive mappings of Banach spaces. Proc. Camb. Philos. Soc. 1964, 60, 439-447. [CrossRef]

20. Aydi, H.; Banković, R.; Mitrović, I.; Nazam, M. Nemytzki-Edelstein-Meir-Keeler type results in $b$-metric spaces. Discret. Dyn. Nat. Soc. 2018, 4745764. [CrossRef]

21. Bailey, D.F. Some theorems on contractive mappings. J. Lond. Math. Soc. 1966, 41, 101-106. [CrossRef]

(C) 2020 by the authors. Licensee MDPI, Basel, Switzerland. This article is an open access article distributed under the terms and conditions of the Creative Commons Attribution (CC BY) license (http://creativecommons.org/licenses/by/4.0/). 